\title{
Community structure, distribution pattern, and influencing factors of soil Archaea in the construction area of a large-scale photovoltaic power station
}

\section{Wei Wu}

Xi'an University of Technology

bo yuan ( $\square$ yuanbo_xut@163.com )

Xi'an University of Technology https://orcid.org/0000-0001-7035-7257

Shengjuan Yue

Xi'an University of Technology

\section{Penghui Zou}

State Power Investment Corporation Ltd: SPIC

\section{Ruoting Yang}

State Power Investment Corporation Ltd: SPIC

Xiaode Zhou

Xi'an University of Technology

\section{Research Article}

Keywords: soil archaeal communities, photovoltaic industrial park, desert ecosystem, high-throughput sequencing

Posted Date: August 20th, 2021

DOI: https://doi.org/10.21203/rs.3.rs-810369/v1

License: (c) (1) This work is licensed under a Creative Commons Attribution 4.0 International License. Read Full License 


\section{Community structure, distribution pattern, and influencing}

\section{factors of soil Archaea in the construction area of a large-scale}

\section{photovoltaic power station}

Wei Wu ${ }^{1,+}$, Bo Yuan ${ }^{1,+, ~ *}$, Shengjuan Yue ${ }^{1,2}$, Penghui Zou ${ }^{3}$, Ruoting Yang ${ }^{3}$ and Xiaode Zhou ${ }^{1}$ (1.Xi'an University of Technology, State Key Laboratory of Eco-hydraulics in Northwest Arid Region, Xi'an 710048, P.R. China)

(2.Qinghai University, Institute of Water Resources and Electric Power, Xining 810016, P.R. China) (3.State Power Investment Group Qinghai Photovoltaic Industry Innovation Center Co., Ltd, Xining 810003, P.R. China)

* Correspondence: yuanbo_xut@163.com (B.Y.)

+ These authors contributed equally to this work. 
Abstract: The photovoltaic power station in Qinghai has been built for 8 years, however, its impact on the regional soil ecological environment has not been studied in depth. To reveal the structure and distribution pattern of archaeal communities in desert soil under the influence of a large photovoltaic power station, a comparative study was carried out between the soil affected by photovoltaic panels and the bare land samples outside the photovoltaic station in Gonghe, Qinghai Province. The abundance, community structure, diversity, and distribution characteristics of archaea were analyzed by quantitative PCR and Illumina-MiSeq high-throughput sequencing, and the main environmental factors affecting the variation of soil archaeal community were identified by RDA. The contribution rate of environmental factors and human factors to microbial community diversity was quantitatively evaluated by VPA. The results showed that there was no significant difference in soil nutrients and other physicochemical factors between the photovoltaic power station and bare land. Thaumarchaeota was the dominant archaeal phylum in the area, accounting for more than $99 \%$ of archaeal phylum, while at the level of genus, Nitrososphaera was the dominant archaeal genera. There was no significant difference in archaeal community structure between and under different types of PV panels. The analysis has shown that the construction of a photovoltaic station has little effect on the community structure of soil archaea in a desert area, and it was speculated that the selection of niche played a leading role in the distribution pattern of soil archaeal community. This study provides the basis for a scientific understanding of the characteristics and distribution patterns of soil archaeal communities affected by the construction of a photovoltaic power station.

Keywords: soil archaeal communities; photovoltaic industrial park; desert ecosystem; high-throughput sequencing

\section{Introduction}

Energy production is considered a major challenge in industrial development (Pires et al. 2012)especially in the context of exponential growth of the global population. Considering the impoverishment and depletion rate of conventional fossil energy sources, several environmentally friendly and efficient renewable energy sources have been investigated recently (Tawalbeh et al. 2021). Among the many renewable energy technologies, solar photovoltaic (PV) technology has become an increasingly important energy supply option worldwide owing to its significant advantages in mitigating climate change, reducing greenhouse gas emissions, and increasing the security of energy supply (Ebhota and Jen 2020; Nižetić et al. 2018; Turney and Fthenakis 2011; Wilberforce et al. 2019). However, based on the product life-cycle theory, the development of any industry and technology is a "doubleedged sword." Solar PVs are no exception, and their positive and negative feedback effects on the ecological environment are gradually emerging (Tawalbeh et al. 2021; Turney and Fthenakis 2011).

Archaea are one of the three domains of life, whose survival was once thought to be restricted to extreme environments. However, the archaeal tree has expanded rapidly with the discovery of new archaeal lineages at different taxonomic levels (Auguet et al. 2010; Bates et al. 2011; Huang et al. 2019; Shi et al. 2016). An increasing number of studies in different environmental systems have shown that archaea are widely distributed in a variety of ecosystems (Anish et al. 2015; Auguet et al. 2012; Bates et al. 2011; Brochier-Armanet et al. 2008; Hu et al. 2016; Jiang et al. 2007)and exhibit significant environmental heterogeneity. Meanwhile, archaea play an important role in mediating biogeochemical processes such as ammonia oxidation (Dai et al. 2018), sulfate reduction (Sorokin et al. 2017), organic matter decomposition (Meng et al. 2019), and greenhouse gas regulation (Liu et al. 2019). Despite the significant impact of archaea on the ecological restoration of soil function, previous studies have focused on bacteria and fungi, and studies on archaeal community composition and function have rarely been considered owing to technical limitations. In addition, various environmental factors including $\mathrm{pH}$, salinity, soil moisture, heavy metals, nutrient levels, vegetation type, and local climatic characteristics (Huang et al. 2019; Meng et al. 2019)significant effects on the composition of soil archaeal communities. In contrast, the variation in soil archaeal communities caused by anthropogenic disturbances such as soil reclamation, improvement, restoration, and engineering construction has received substantially less attention. Limited studies have suggested that anthropogenic disturbances might be an important factor influencing the composition (Shi et al. 2016; Wang et al. 2011)and taxon abundance of archaeal communities.

As a typical representative of the arid desert region in Northwest China, Talatan, where the Qinghai Gonghe Photovoltaic Industrial Park is located, is an ideal area for the development of solar PV power generation because 
of its poor soil physical structure, low soil nutrient and organic matter content, high salinity, aridity and water scarcity, extreme temperatures and dryness, strong winds, and strong UV radiation. Although soil archaea are among the most diverse and abundant organisms in the soil, microbial development and activities are disturbed in such a nutrient-limited environment, which affects the biogeochemical cycling of nutrients (Hu et al. 2019b; Shi et al. 2016; Wang et al. 2015a; Wei et al. 2020). This makes the ecosystem more sensitive to disturbance, slow to recover from disturbance, and vulnerable to climate change. The increasing number of crystalline PV panels in the Qinghai Gonghe Photovoltaic Industrial Park has impacted the ecological environment of the region. Current studies mainly elucidated the ecological and environmental effects of climate, vegetation, and soil from a macroscopic perspective (Gao et al. 2016; Li et al. 2019; Li et al. 2016), and no research on the microscopic level of soil archaeal communities has been reported.

Based on the abovementioned background, this study used quantitative PCR and 16S rRNA high-throughput sequencing techniques to analyze the number and diversity of soil archaea in PV power plants. This study also investigated the structure, number, diversity, and distribution characteristics of soil bacterial communities in large photovoltaic power plants and elucidated the effects of environmental and anthropogenic factors on the distribution patterns of soil archaeal communities. This is the first study to investigate the composition and structural characteristics of soil archaeal communities under the influence of PV plant construction. It will provide a theoretical basis for the comprehensive assessment of the ecological and environmental effects of PV parks in the future.

\section{Materials and methods}

Study area and sample collection. The Gonghe Photovoltaic Industrial Park $\left(98^{\circ} 54^{\prime} \mathrm{E}-101^{\circ} 22^{\prime} \mathrm{E}, 35^{\circ} 46^{\prime} \mathrm{N}-\right.$ $37^{\circ} 10^{\prime} \mathrm{N}$ ) is in Talatan, Gonghe County, Qinghai Province (Fig. 1), with an altitude of 2900-3100 m. Gonghe County has a plateau subtropical climate, with average annual evaporation and precipitation of $1800 \mathrm{~mm}$ and $303 \mathrm{~mm}$, respectively, the sunlight of $2772 \mathrm{~h}$, the temperature of $4.1^{\circ} \mathrm{C}$, and solar radiation of $6564.26 \mathrm{MJ} / \mathrm{m}^{2}$. Due to strong wind erosion, low precipitation, and overgrazing, there is serious vegetation degradation in Talatan, and the degree of soil sanding and desertification is increasing yearly. At present, the park is divided into new energy generation (PV power park and wind power park) and industrial processing and manufacturing areas. The total area is 3,028.5 $\mathrm{km}^{2}$, including a $609.6 \mathrm{~km}^{2} \mathrm{PV}$ power park, $2,400 \mathrm{~km}^{2}$ wind power park, and $18.9 \mathrm{~km}^{2}$ industrial processing and manufacturing area.
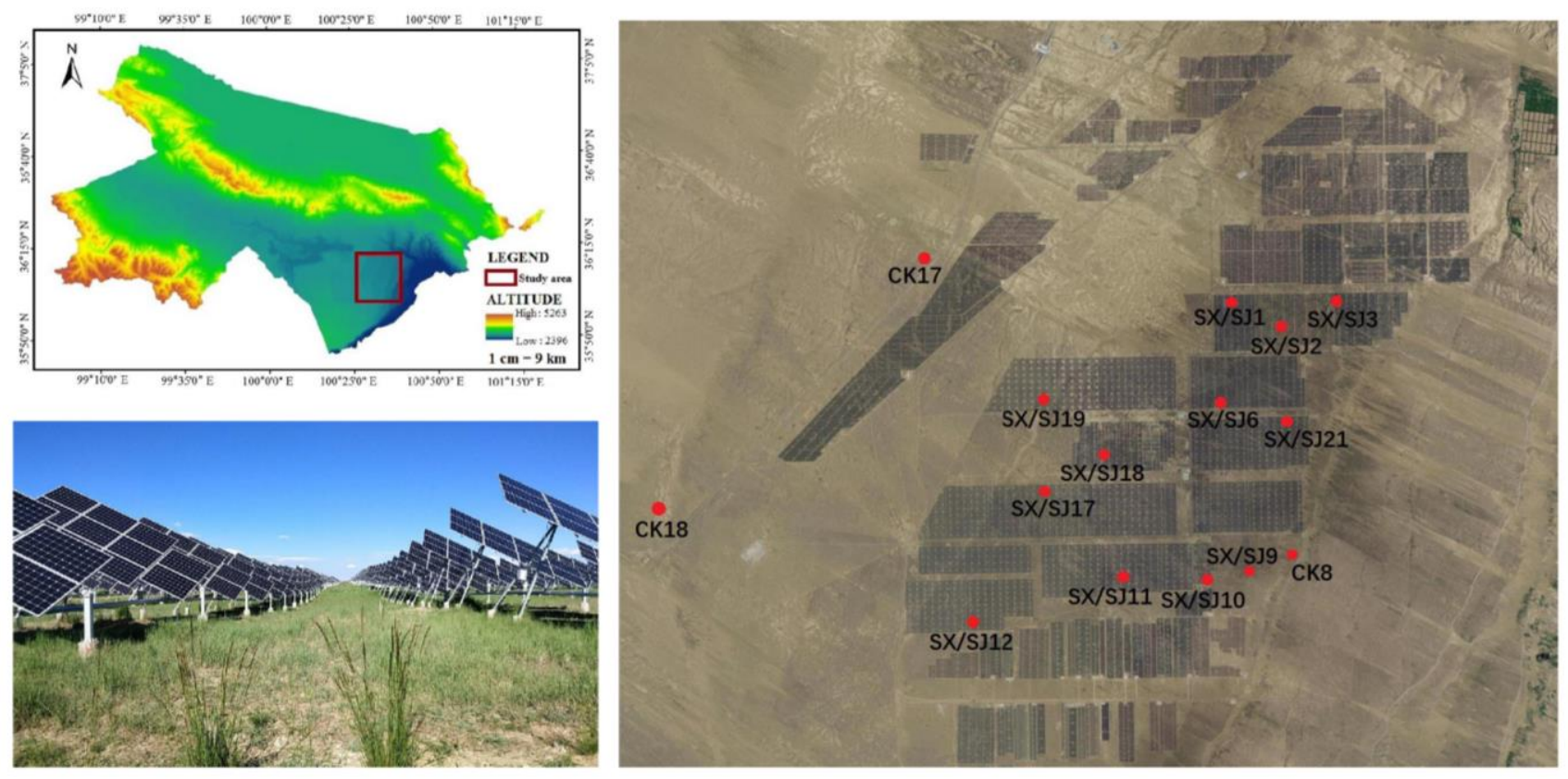

Fig. 1 Location of the sampling sites in photovoltaic industrial park of Gonghe, Qinghai.

In mid-August 2020, a total of 27 representative soil samples were collected from the PV park, as shown in Table. S1. GPS (UG802, Tibbo, China) was used to locate the sites throughout the sampling process, and the latitude, 
longitude, and elevation information of the sites were recorded. The sampling points were divided into three groups: flat single axis (FSA, sampling number 1, 10), oblique single axis (OSA, sampling number 2, 3, 9), and fixed axis (FIX, sampling number $6,11,12,17,18,19,21)$ according to the orientation of the PV tracking brackets. For each group, samples were collected at the sampling points under the PV stand panels (SX) and between the panels (SJ). To distinguish between the characteristics of soil archaea at sample sites with and without PV stand panels, three control sampling sites (CK) were set up on the bare ground outside the PV power plant. Five independent surface soil samples (sampling depth: 0-10 cm) were collected separately from each sampling site $(2 \mathrm{~m} \times 2 \mathrm{~m}$ sample square $)$ and mixed into one composite sample. Samples for characterizing the physical and chemical properties of the soil were packed in sterile polyethylene bags, transported to the laboratory, air-dried, sieved through a $1 \mathrm{~mm}$ screen to remove debris and plant roots, and stored. Samples for microbiological analysis were packed in $50 \mathrm{~mL}$ sterile polyethylene conical centrifuge tubes, stored in liquid nitrogen, rapidly transported back to the laboratory, and stored at $-80{ }^{\circ} \mathrm{C}$ in a freezer.

Soil physicochemical parameters and vegetation diversity analysis. Soil samples were dried at $65{ }^{\circ} \mathrm{C}$ and analyzed for chemical properties according to standard methods. Soil water content was determined using the weight method, soil pH, and conductivity (FE30, Mettler-Toledo, Switzerland) at a soil-to-water ratio of 1:2.5. Soil organic matter (SOM) and soil organic carbon (SOC) were determined by the dichromate oxidation method (Nelson et al. 1982). Total nitrogen (TN) was determined by the dry combustion method in an elemental analyzer (Elementar Vario Macro cube, Hanau, Germany). The soil carbon-to-nitrogen ratio was calculated from OC and TN. Alkaline nitrogen was determined using a Kjeldahl distillation system (C. Gerhardt, Germany). The soil total phosphorus (TP) and total potassium content was determined using the perchloric acid digestion method Nelson and Sommers (1973). Soil alkaline nitrogen, fast-acting potassium, and fast-acting phosphorus content was determined by visibleNIR spectroscopy ( $\mathrm{Li}$ et al. 2007). The vegetation survey covered the entire study area, and the plant community species diversity index was calculated (unpublished data).

High-throughput sequencing and fluorescent quantitative polymerase chain reaction. Total DNA was extracted from soil archaea using a Fast DNA Spin Kit (MP Biomedicals, USA) and stored at $-80^{\circ} \mathrm{C}$. The quality of extracted DNA was determined by $1.0 \%$ agarose gel electrophoresis. Polymerase chain reaction (PCR) amplification was performed on the V4-V5 region of the archaeal 16S r RNA gene, and the primers used for PCR amplification were Arch524F (5'-TGYCAGCCGCCGCGGTAA-3') and Arch958R (5'-YCCGGCGTTGAVTCCAATT-3') (Pires et al. 2012). The PCR amplification system consisted of a $2.5 \mu \mathrm{L} 10 \times$ PCR buffer, $3 \mathrm{mM} \mathrm{MgCl}, 160 \mu \mathrm{M}$ dNTPs, $100 \mathrm{pmol}$ of upstream primer, $100 \mathrm{pmol}$ of downstream primer, $0.625 \mathrm{U}$ Taq polymerase, and $2 \mu \mathrm{L}$ DNA template (1:20 dilution of total DNA). The PCR amplification reaction protocol consisted of initial denaturation at $95{ }^{\circ} \mathrm{C}$ for $5 \mathrm{~min}, 35$ cycles of denaturation at $95{ }^{\circ} \mathrm{C}$ for $1 \mathrm{~min}$, annealing at $56^{\circ} \mathrm{C}$ for $30 \mathrm{sec}$, and extension at $72{ }^{\circ} \mathrm{C}$ for 1.5 min, followed by final extension at $72{ }^{\circ} \mathrm{C}$ for $10 \mathrm{~min}$. The PCR products were separated by electrophoresis on $2 \%$ agarose gels, and gel bands containing target fragments were cut and recovered with a gel extraction kit (AXYGEN, USA). The PCR products were quantified using a fluorescence microplate reader (BioTek, FLx800, USA) with the Quant-iT PicoGreen dsDNA Assay Kit (ThermoFisher, USA). Based on the quantification results, samples were pooled in the appropriate ratio according to the required read depth. Sequencing libraries were prepared using an Illumina TruSeq Nano DNA LT Library Prep Kit. After the samples passed quality check, paired-end sequencing with read-length of 250 bps was performed on an Illumina sequencer by Shanghai Personal Biotechnology Co., Ltd.

The archaeal 16S rRNA gene was quantified using an AceQ qPCR SYBR Green Master Mix on a CFX96 Touch model real-time fluorescent quantitative PCR instrument (Bio-Rad, USA). Amplification primers were consistent with high-throughput sequencing primers. The total reaction volume was $15 \mu \mathrm{L}$, consisting of $12.5 \mu \mathrm{L}$ of AceQ qPCR SYBR Green Master Mix (2X), $0.7 \mu \mathrm{L}$ of upstream and downstream primers, $1 \mu \mathrm{L}$ of DNA template, and $15 \mu \mathrm{L}$ of $\mathrm{ddH}_{2} \mathrm{O}$. The reaction protocol consisted of initial denaturation at $95{ }^{\circ} \mathrm{C}$ for 5 min, followed by 40 cycles of denaturation at $95^{\circ} \mathrm{C}$ for $10 \mathrm{sec}$, and annealing and extension at $60{ }^{\circ} \mathrm{C}$ for $60 \mathrm{sec}$.

All sequence data from Illumina MiSeq sequencing in this study have been submitted to the public NCBI database (http://www.ncbi.nlm.nih.gov/) under the accession number PRJNA690680. 
Bioinformatics data processing and analysis. The raw data from Illumina high-throughput sequencing was first demultiplexed using the barcodes of all libraries. Quality control filtering was performed based on read quality. Paired reads were then merged into one sequence based on the overlap of the reads. Finally, high quality sequences for each sample were obtained by demultiplexing with barcode and primer sequences, and sequence orientation was corrected according to the forward and reverse barcodes and primer orientation. Chimeras were removed using a combination of de novo and reference-based methods (Usearch software and Gold database). QIIME (version 1.8.0, http://drive5.com/uparse/) was used. Operational Taxonomic Units (OTUs) were grouped and sorted according to a $97 \%$ similarity level. OTUs with abundance $<0.01 \%$ of the total sequencing depth were removed. The sequence with the highest abundance in each OTU was selected as the OTU representative sequence. Each OTU representative sequence was then compared with the Greengenes database (Luvizott et al. 2019)to obtain the annotation proportion and relative abundance of species of each taxonomic rank of OTUs. The RDP classifier Bayesian algorithm was used to taxonomically analyze the OTU representative sequences at $97 \%$ similarity level, and QIIME software (version 1.8.0) was used to obtain the abundance distribution table for each sample at five taxonomic levels including Phylum, Class, Order, Family, and Genus.

Alpha diversity was calculated using the vegan package and OTU table package in R software (version 3.5.1), and the Kruskal-Wallis test was used to compare the differences in diversity between different subgroups. Nonmetric multidimensional scaling analysis (NMDS), analysis of similarity (ANOSIM), and multivariate analysis of variance (ADONIS) were performed using different functions in the vegan package to test the significance of differences in the structure of archaeal communities in soils. Hierarchical cluster analysis (beta diversity) based on Bray-Curtis distance was performed on soil samples. To estimate the relative importance of environmental and anthropogenic factors in the formation of community composition, variance decomposition analysis (VPA) was performed using (partially) constrained principal coordinate analysis (CAP) using the varPart function in the vegan package in R software based on Bray-Curtis dissimilarity (Rui et al. 2015). Spearman's rank correlation analysis was performed using SPSS 22.0 to test the relationship between archaeal abundance and diversity and different environmental variables. In addition, redundancy analysis (RDA) was performed using CANOCO 5.0 to reveal the correlation between archaeal community composition and environmental variables.

\section{Results}

Spatial variation characteristics of soil physicochemical factors. Soil physicochemical characteristics were analyzed for three types of sample plots and three different PV stand sampling sites, respectively (Fig. 2). The soils in the study area were semi-arid alkaline $(\mathrm{pH}=8.41-9.60)$ with water content ranging from $4.5 \%$ to $26.25 \%$. Soil $\mathrm{pH}$ and water content were both higher inside the PV stand sites than outside but did not show significant differences. Soil temperature was significantly lower in the field than in the bare ground outside the field during the sampling period due to shading by PV panels. TN and TC in the soil samples did not show statistically significant differences ( > 0.05), were highest in the samples under the PV stand panels and did not show a significant pattern between different PV tracking stands. Soil OM and OC content was the highest in the inter-panels sampling sites and the lowest in the off-site sampling sites; this was also the highest in the flat uniaxial sampling sites and the lowest in the oblique uniaxial sampling sites, with no significant differences among sampling sites $(P>0.05)$. TP and potassium content did not differ significantly $(P>0.05)$ among sampling sites. Soil alkaline nitrogen and fast-acting phosphorus did not differ significantly $(P>0.05)$ among sampling sites, and fast-acting potassium was the highest under the flat single-axis PV panels and the lowest on the bare ground outside the field. 

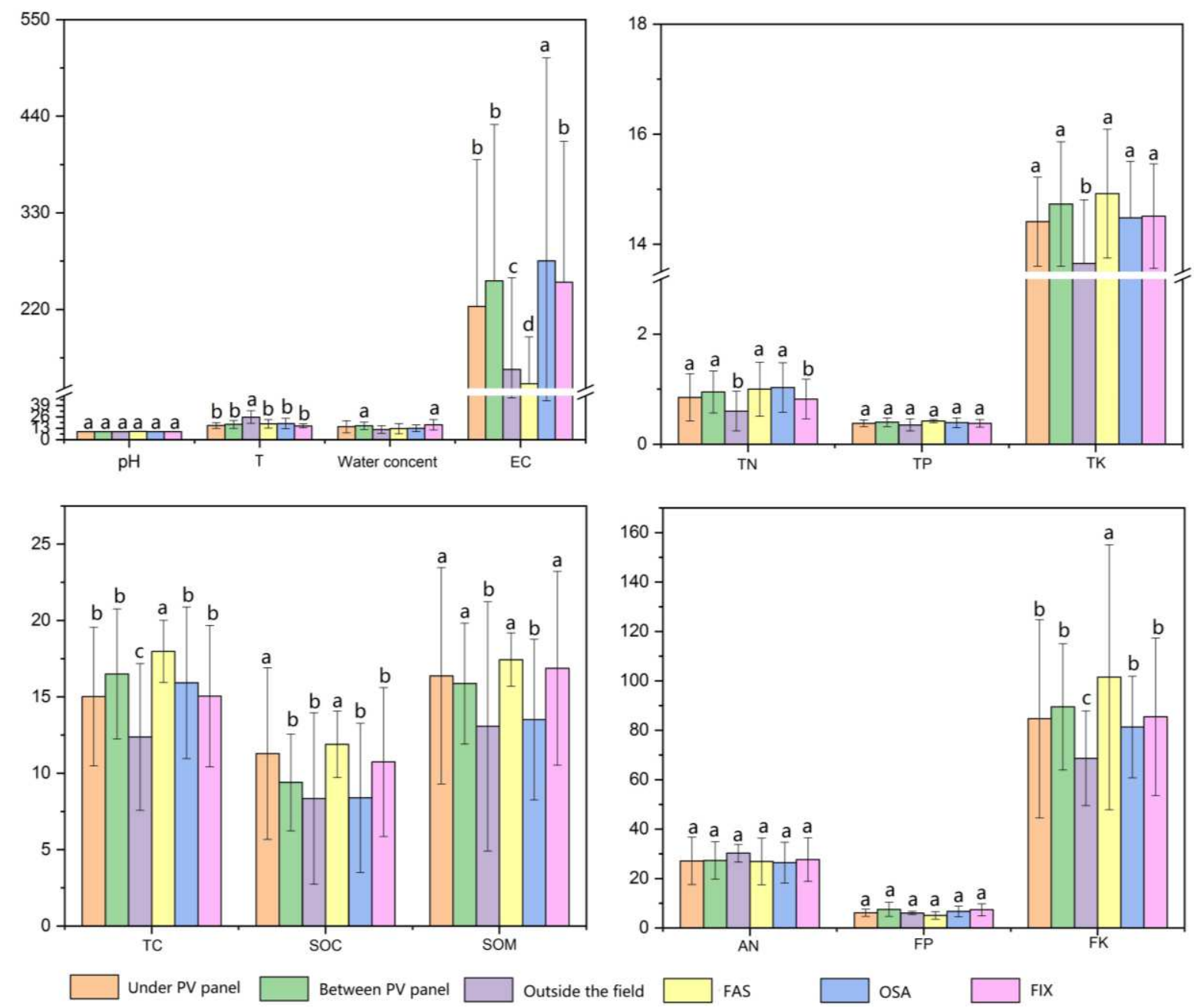

Fig. 2. Physicochemical parameters of the soil samples in photovoltaic industrial park of Gonghe, Qinghai. FSA, flat single-axis; OSA, oblique single-axis; FIX, fixed trackers; Temp., water temperature; EC, electrical conductivity; TN, total nitrogen; TC, total carbon; TP, total phosphorus; TK, total potassium; AN, alkalinehydrolysable nitrogen; FP, fast-acting phosphorus; FK, fast-acting potassium; SOC, soil organic carbon; SOM, soil organic matter. Different letters after the columns indicate significant differences $(P<0.05)$.

Abundance of soil archaeal. A total of 1,722,647 valid reads were obtained by high-throughput sequencing of different soil archaea 16S rRNAs in the PV park, and the number of valid high-quality reads of archaea ranged from 50,730 to 91,564 (Table S2). The corresponding library coverage rate ranged from $80.9 \%$ to $89.8 \%$, indicating that the libraries of samples in this study contained most archaea taxa and basically reflected the structural composition of the endophytic Archaea community in the samples. As shown in Fig. 3, the number of archaeal 16S rRNA genes per gram of soil (dry weight) under the PV tracker panels ranged from $2.30 \times 10^{9}$ to $5.01 \times 10^{10}$ copies, with a mean value of $2.23 \times 10^{10}$ copies. The number of archaeal $16 \mathrm{~S}$ rRNA genes per gram of soil (dry weight) between the panels ranged from $3.84 \times 10^{9}$ to $6.37 \times 10^{10}$ copies, with a mean value of $2.23 \times 10^{10}$ copies. In the bare ground outside the PV park, the number of archaeal $16 \mathrm{~S}$ rRNA genes per gram of soil (dry weight) ranged from $2.25 \times 10^{10}$ to $8.37 \times 10^{10}$ copies, with a mean value of $4.39 \times 10^{10}$ copies. In the PV park area, the number of archaeal $16 \mathrm{~S}$ rRNA genes per gram of soil (dry weight) ranged from $2.25 \times 10^{10}$ to $8.37 \times 10^{10}$ copies, with a mean value of 4.39 $\times 10^{10}$ copies. The number of soil archaea was higher under the panels than between the panels except sampling points S1, S2, S6, S10, S17, and S21 in the PV park, where the number of soil archaea between the panels was higher. The number of archaea was higher between the plates than under the plates at all the sampling points of flat single-axis tracking bracket PV panels (S1 and S10). The number of archaea between the plates and under the plates did not have clear pattern at the sampling points of oblique single-axis tracking bracket PV panels (S2, S3, and S9) 
and fixed tracking bracket sampling points (S6, S11, S12, S17, S18, S19, and S21). The number of archaea per gram of soil (dry weight) was not spatially significantly different $(P>0.05)$ among the sampling sites within the PV plant.

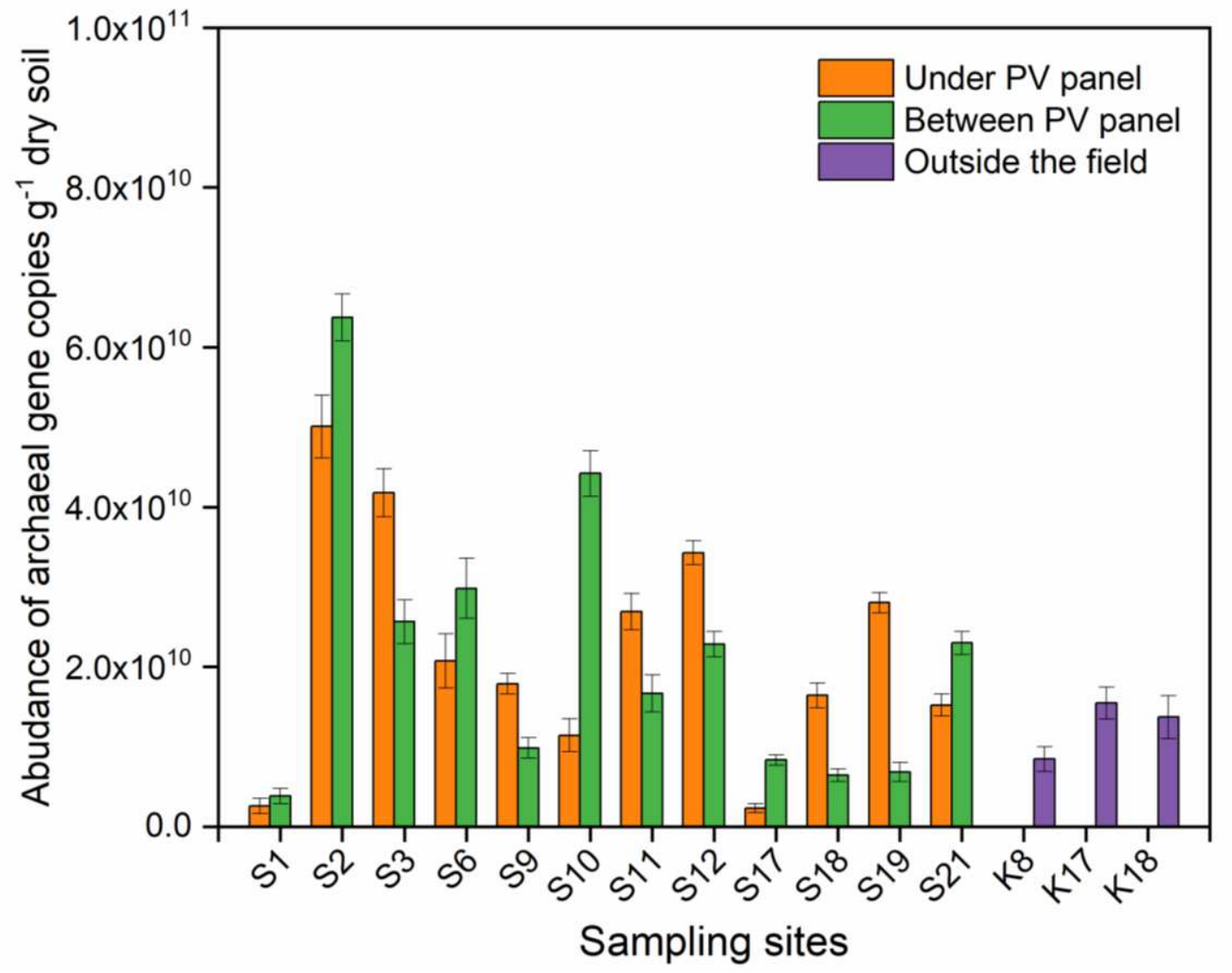

Fig. 3 Abundance of archaeal community in the soils of Gonghe photovoltaic power station, Qinghai.

Soil archaea alpha diversity. The alpha diversity of soil archaea was not significantly different $(P>0.05)$ between the under-panels (INX), inter-panels (INJ), and off-site (OUT) sampling sites in the PV plant, as shown in Fig. 4. The Chaol index and observed species index indicated that soil microbial diversity and richness were slightly higher in the INX and INJ samples than in the OUT samples but not significantly different $(P>0.05)$. The overall trend of the Shannon and Simpson index were inconsistent among the 27 samples. The Shannon index showed slightly lower species diversity and evenness at the INJ) and INX sites than at the OUT sites, again not significantly different $(P>$ 0.05 ) and with little fluctuation at each site. The Faith's PD index showed that the evolutionary diversity at the OUT sites was significantly lower than at INX and INJ sites. The Pielou's evenness index indicated that the species evenness of the INJ sample site was slightly lower than those of the INX and OUT sample sites. The Good's coverage index of the three types of sampling sites indicated that the species diversity index of the OUT sampling sites was relatively lower. 

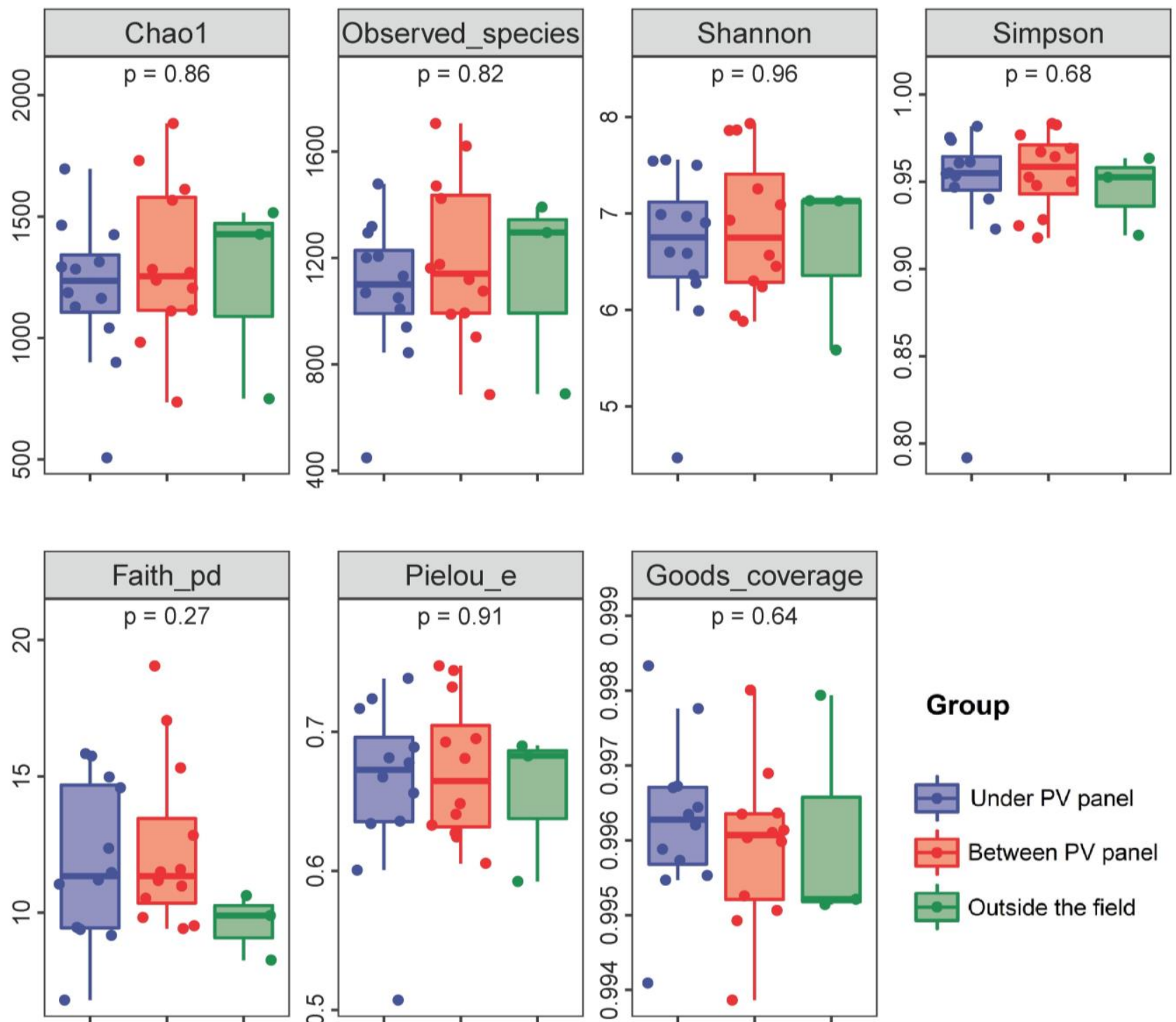

\section{Group}
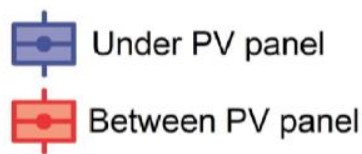

Outside the field

Fig. 4 Alpha diversity of archaeal community among different sample groups.

\subsection{Structural characteristics and beta-diversity of archaeal communities}

The relative abundance of soil archaea species at the taxonomic level of Phylum and Genus in the PV park of Qinghai Gonghe Photovoltaic Industrial Park is shown in Fig. 5(a,b). The soil archaeal community in the study area was structurally homogeneous. Analysis of archaeal phylum or candidate phylum with a relative proportion $>0.1 \%$ showed a total of two phyla, among which Thaumarchaeota (99.84\%) dominated and was widely distributed in all sampling sites, while Euryarchaeota $(0.14 \%)$ was only marginally distributed in some sampling sites. The structure of the archaeal community in the 27 soil samples was similar, with the proportions of dominating Thaumarchaeota ranging from $98.46 \%$ to $100 \%$. For different types of PV panels (fixed-axis, flat-axis, and oblique-axis), the abundance of Thaumarchaeota was not significantly different $(P>0.05)$ in the INX and INJ soil samples. Similarly, Nitrososphaera was the dominant genus of archaea with relative proportions of $98.44-100.00 \%$ (mean value $99.82 \%$ ). 
(a)

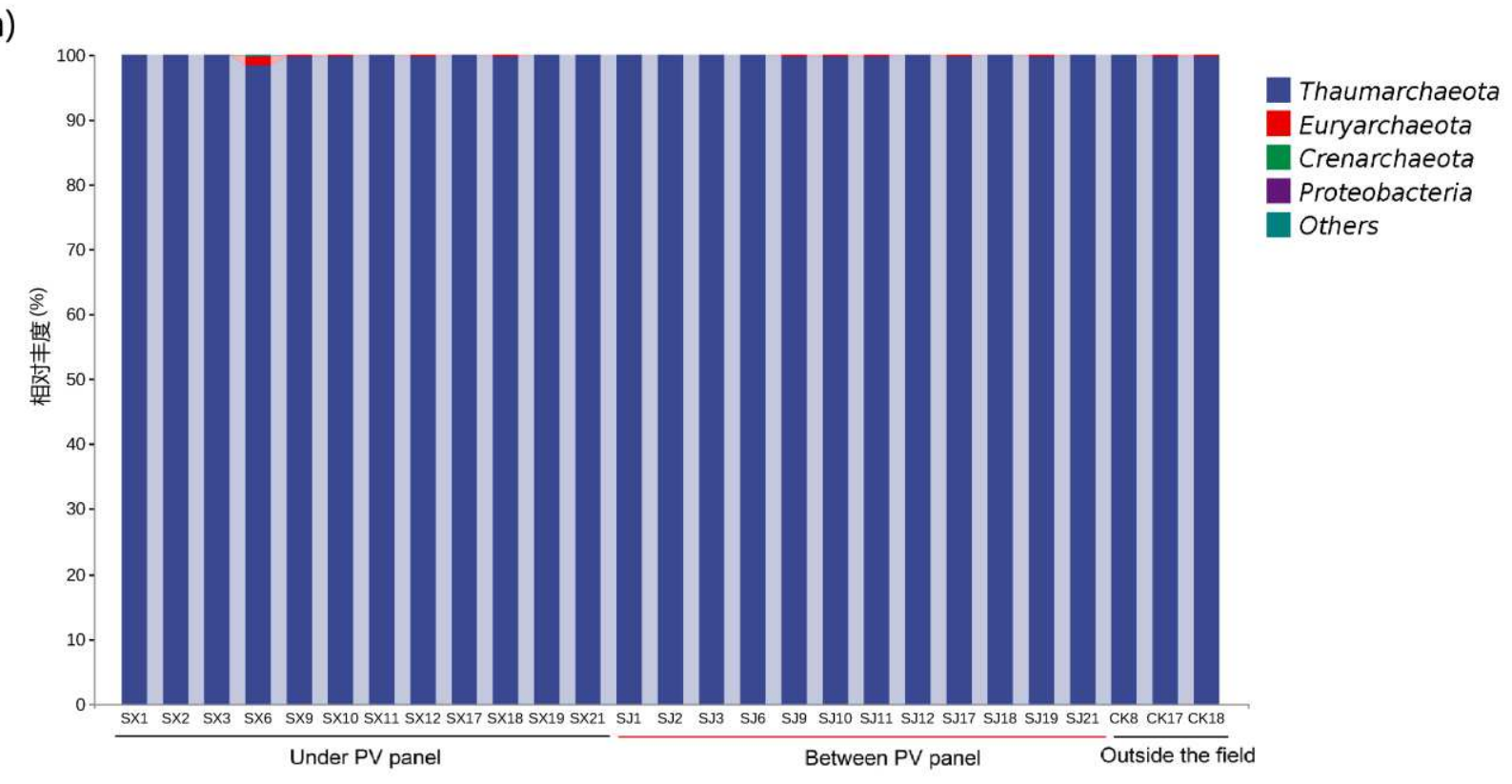

(b)

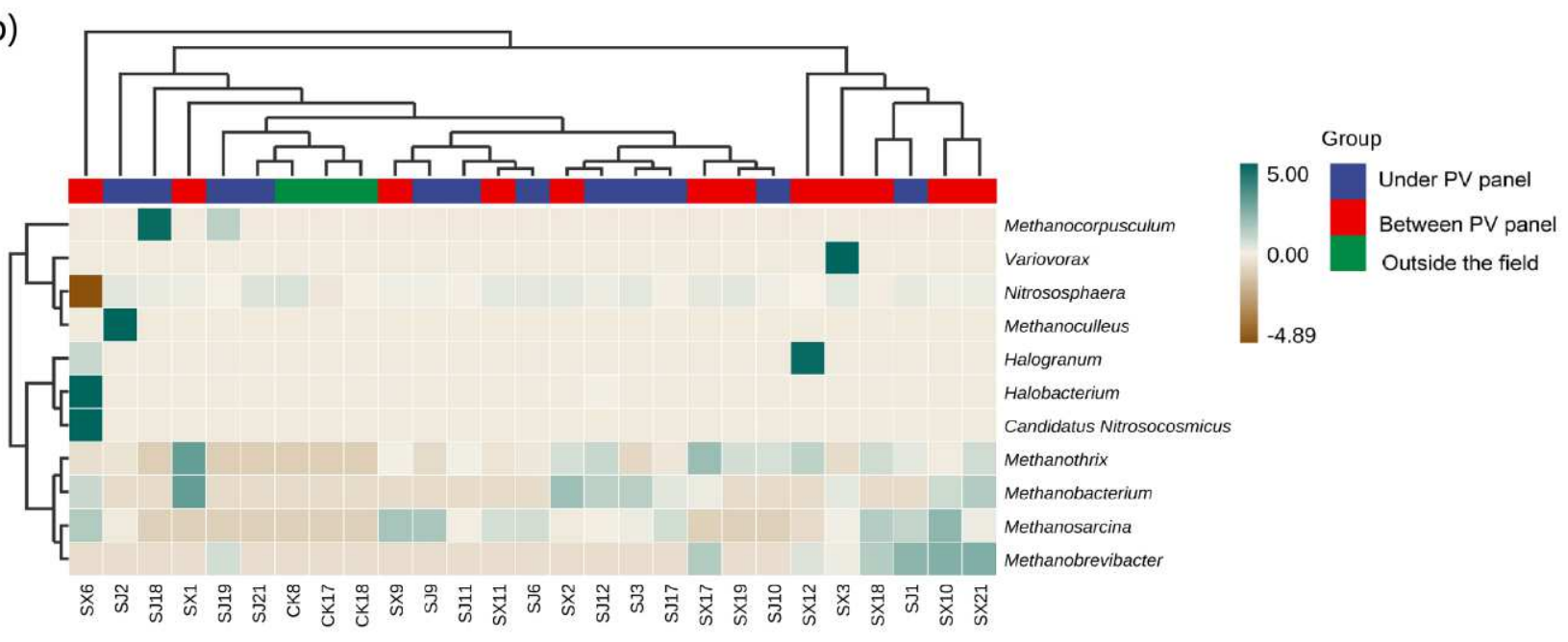

Fig. 5 Relative distribution of phylum and genes soil archaeal community in different sampling sites.

As shown in Fig. 6(a, b, c), the stress values of NMDS analysis for soil bacterial communities in different groups of sampling sites were 0.125 and 0.045 , respectively, which indicated that the ranking results in this study reflected the differences of soil archaeal communities in different sampling sites. Based on the Bray-Curtis distance algorithm (Table S3), no significant variation $(P>0.05)$ in soil archaeal communities was observed in the three types of fields, which was confirmed by ANOSIM $(\mathrm{R}=0.03, \mathrm{P}=0.26)$ and ADONIS $(\mathrm{R}=0.25, \mathrm{P}=0.53)$. The statistical results from the weighted UniFrac (ANOSIM: $\mathrm{R}=0.01, \mathrm{P}=0.35$; ADONIS: $\mathrm{R}=0.24, \mathrm{P}=0.59$ ) and unweighted UniFrac distance (ANOSIM: $\mathrm{R}=$ $0.02, \mathrm{P}=0.34$; ADONIS: $\mathrm{R}=0.32, \mathrm{P}=0.12$ ) were consistent with the results from the Bray-Curtis distance. The Mantel tests did not show significant correlation between Bray-Curtis and unweighted UniFrac distances (Mantel $\mathrm{R}=0.14, P>$ 0.05 with 5,000 permutations), Bray-Curtis and weighted UniFrac distances (Mantel $\mathrm{R}=0.17, P>0.05$ with 5,000 permutations), and unweighted and weighted UniFrac distances (Mantel $\mathrm{R}=0.128, P>0.05$ with 5,000 permutations). 

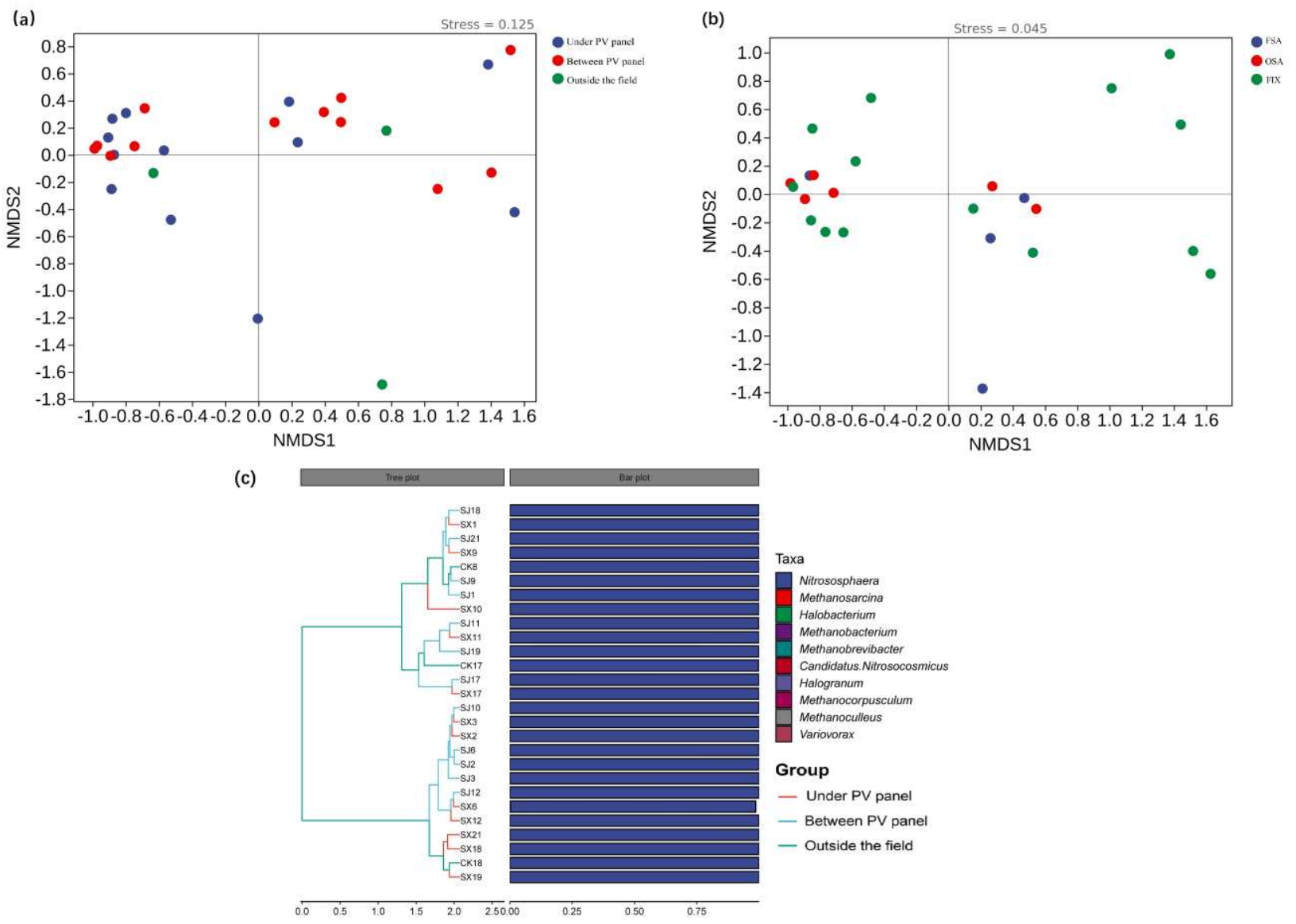

Fig. 6 NMDS (a, b) and Hierarchical Cluster analysis of archaeal community compositions (top 10 archaeal genera(c))based on the relative abundance.

To investigate the microbial community characteristics and functions in the soil archaeal communities of the PV field, the archaeal genera whose relative proportions reached $0.1 \%$ in each sample site were screened. Cluster analysis was performed on the genera that were picked up from the screening using the Bray-Curtis algorithm. As shown in Fig. 6(c), the archaeal community composition of the sampling sites formed two major branches according to the regional soil geographic environmental conditions and the deployment of PV tracking support panels. The sampling sites S2, S3, S6, S12 (containing INX and INJ), and CK18 clustered into one group. S1, S9, S17 (containing INX and INJ), CK8, and CK17 clustered into another group, and the remaining sampling sites were randomly distributed. Similar to the UPGMA cluster analysis of bacteria, the analysis of archaeal communities showed that rather than clustering into groups based on sampling sites or geographical locations, the INX and INJ soil samples showed a random distribution.

\subsection{Environmental drivers of soil archaeal communities and VPA analysis}

As shown in Table 1, Spearman correlation analysis indicated that the number of archaea in the soil had significant positive correlations with soil OM and total OC content $(P<0.01)$. The Chaol index of archaea had a significant negative correlation with soil alkaline nitrogen content $(P<0.01)$. The Faith's phylogenetic diversity index had a highly significant positive correlation with total carbon content $(P<0.01)$. The Shannon index had a highly significant negative correlation with alkaline nitrogen content $(P<0.01)$ and a highly significant positive correlation with total carbon $(P<0.01)$. The Simpson's index had significant positive correlations with total carbon and OM content $(P<0.01)$ and a highly significant negative correlation with alkaline soluble nitrogen $(P<0.01)$. To further investigate the contribution of soil physicochemical factors to soil archaea in the PV park, a ranking analysis was performed using CANOCO 5.0, and the results are shown in Fig. 7. For the archaeal community, the first two axes of the RDA analysis explained $64.82 \%$ and $0.01 \%$ of the archaeal community composition, respectively. For the ranking plot based on the first two RDA ranking axes, soil electrical conductivity $(\mathrm{EC})(\mathrm{F}=11.5, \mathrm{P}=0.028,499$ permutations $)$, alkaline nitrogen $(\mathrm{JN})(\mathrm{F}=4.4, \mathrm{P}=0.03$, 
499 permutations) and water content (WC) $(\mathrm{F}=5.4, \mathrm{P}=0.028,499$ permutations) passed the Monte Carlo significance test, indicating that these three environmental factors can significantly affect the entire archaeal community.

Tab. 1 Spearman correlation analysis of bacterial communities with soil physicochemical parameters

\begin{tabular}{cccccccc}
\hline & Abundance & Chao1 & Faith-pd & Shannon & Simpson & Thaumarchaeota & Nitrososphaera \\
\hline Water concent & 0.291 & 0.036 & 0.066 & 0.09 & -0.006 & $\mathbf{0 . 5 7 8 * *}$ & $\mathbf{0 . 5 7 8 * *}$ \\
pH & 0.046 & 0.232 & -0.111 & 0.058 & -0.028 & -0.041 & -0.041 \\
EC & -0.008 & -0.11 & -0.091 & -0.177 & -0.299 & 0.023 & 0.023 \\
Temp & -0.027 & 0.091 & 0.111 & 0.118 & 0.087 & -0.179 & -0.179 \\
TP & 0.2 & -0.284 & 0.187 & 0.075 & 0.165 & -0.218 & -0.218 \\
TK & 0.112 & -0.13 & 0.167 & 0.058 & 0.146 & 0.245 & 0.245 \\
TN & 0.02 & -0.021 & 0.219 & 0.075 & 0.184 & -0.137 & -0.137 \\
TC & 0.032 & 0.093 & $\mathbf{0 . 4 6 3} * *$ & $\mathbf{0 . 4 2 5 * *}$ & $\mathbf{0 . 4 7 5 * *}$ & 0.242 & 0.242 \\
AN & 0.083 & $\mathbf{- 0 . 3 8 6 *}$ & -0.237 & $-\mathbf{0 . 4 3 5 * *}$ & $\mathbf{- 0 . 4 7 1 * *}$ & -0.251 & -0.251 \\
FK & 0.077 & -0.236 & -0.104 & -0.007 & 0.105 & 0.050 & 0.050 \\
SOC & $\mathbf{0 . 4 0 5 * *}$ & 0.115 & 0.216 & 0.249 & 0.335 & 0.140 & 0.140 \\
SOM & $\mathbf{0 . 4 3 0 * *}$ & 0.044 & 0.31 & 0.298 & $0.379 *$ & 0.170 & 0.170 \\
FP & -0.07 & -0.014 & -0.229 & -0.136 & -0.139 & -0.032 & -0.032 \\
\hline
\end{tabular}

Temp., water temperature; EC, electrical conductivity; TN, total nitrogen; TC, total carbon; TP, total phosphorus; TK, total potassium; AN, alkaline-hydrolysable nitrogen; FP, fast-acting phosphorus; FK, fast-acting potassium; SOC, soil organic carbon; SOM, soil organic matter.

* indicate significant differences $(P<0.05)$; ** indicate significant differences $(P<0.01)$.

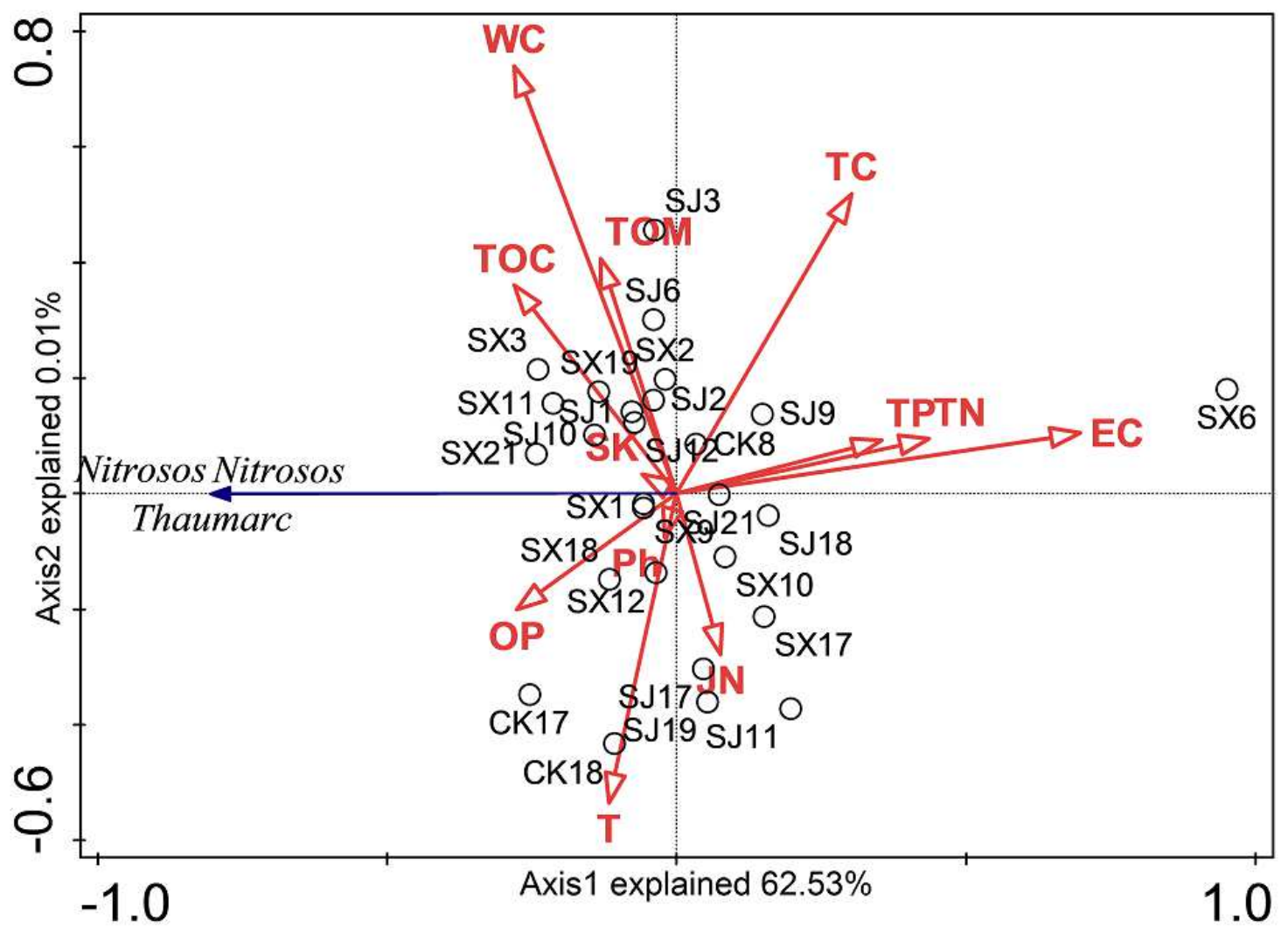

Fig. 7 Redundancy analysis (RDA) between dominant bacterial and the soil properties.

VPA was performed to quantify the relative contribution of different variables that may change the structure of the archaeal community (Fig. 8). Based on the available research data, the variables were divided into three groups: 
soil, vegetation, and anthropogenic influences (PV panel installation). Among them, soil physicochemical characteristics, vegetation growth condition, and PV panel installation parameters explained $33.67 \%, 25.77 \%$, and $7.02 \%$ of the observed variations, respectively, leaving $48.59 \%$ of the variations unexplained (ANOVA, $p=0.001$, Figure 8 ). The PV panel installation parameters alone explained only $0.09 \%$ of the variations, and its contribution was mainly related to plant growth conditions (6.91\%) and soil physicochemical properties (2.56\%). Soil physicochemical properties explained $25.53 \%$ of the variations, among which soil moisture content and electrical conductivity passed the substitution test $(P<0.05)$.

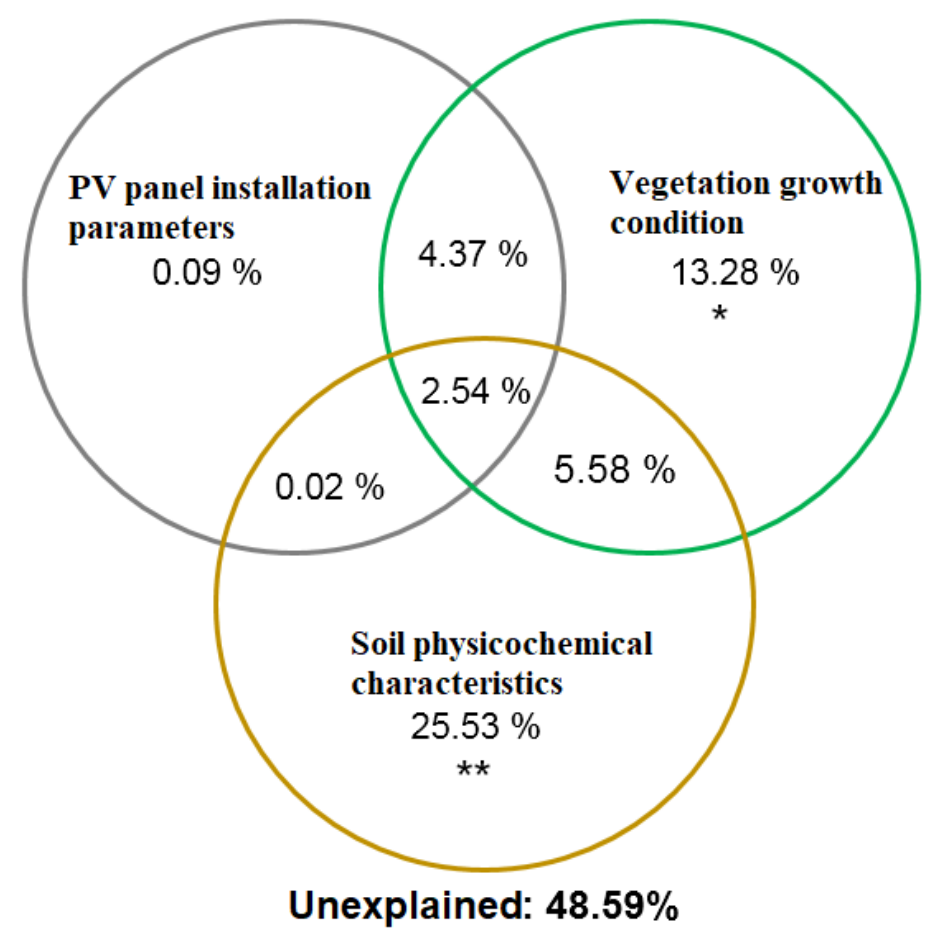

Fig. 8 VPA of soils, plants and PVs factors and archaeal OTUs. The pure effects of spatial and environmental factors were tested for significance with the permutation test.

\section{Discussion}

The Gonghe Photovoltaic Industrial Park is in the semi-arid desert region of Northwest China, and the soil in the park exhibits characteristics of semi-arid conditions, high salinity, and low OM concentrations, providing a unique ecosystem for archaeal communities. In this study, we used 16S rRNA amplicon sequencing to identify archaeal populations in the surface soil collected from specific areas.

Soil archaea abundance. Information on the abundance of archaeal communities in desert soil is still scarce. Spatiotemporally, soil archaebacterial abundance varies considerably among studies. A study of archaea in the Tengger Desert during the vegetation recovery period showed that the 16S rRNA gene copy number of archaeal communities increased significantly during the first 5 a of biological soil crust development, and then decreased with ecological succession (Zhao et al. 2020). However, another study on Tengger Desert soil showed that the abundance of archaeal genes generally increased throughout the succession after 61 a of vegetation restoration (Hu et al. 2019b). The results of several previous studies have also shown that soil microbial communities can increase rapidly in the early stages of desert vegetation restoration (Liu et al. 2013). In the present study, the abundance of soil archaeal communities at the PV farm was approximately 1,000 times higher than that of soils in the early stages of vegetation recovery in the Tengger Desert, which may be related to the short construction time of the PV farm (8 a) and the fact that the vegetation in the PV park was in the early stages of recovery. The unique desert soil recovery habitat in which the park is located may also affect archaeal abundance. However, our study was unable to track the abundance of archaea in the same areas prior to construction of the PV park, and future studies should focus on historical data and spatiotemporal relationships. 
It is commonly assumed that the placement of solar PV panels plays an important role in reducing external environmental stresses such as wind and heat, enhancing the level of development of shrubs and herbaceous plants under and between the panels and promoting nutrient and energy acquisition by microorganisms; therefore, the abundance of soil microorganisms will increase significantly within the PV park area. However, our study on soil archaea showed the opposite results, with a decreasing trend in archaeal abundance within the PV park $\left(2.20 \times 10^{10}\right)$ compared with the bare ground outside the PV park (mean value: $4.39 \times 10^{10}$ ). The most likely reason is that archaea have formed a specific ecological succession pattern and ecological niche under the extreme environment. They have strong adaptability to the poor soil texture and nutrient environment and can effectively use the limited nutrients and low moisture in the desert ecosystem for growth and reproduction. The construction of the PV site broke the original ecological succession pattern and resource utilization sequence. While there was competition among bacterial, fungal, and archaeal communities (Feng et al. 2017; Rothrock and Garcia-Pichel 2005)may be at a disadvantage in competition with bacteria or fungi (Bates et al. 2011; Zhao et al. 2020). To the best of the authors' knowledge, this study is the first to reveal differences in the abundance of soil archaea under the influence of different types of PV stands.

Soil archaeal abundance is closely related to $\mathrm{OC}$ and $\mathrm{OM}$, which suggests that $\mathrm{OM}$ and the most basic metabolic source of archaea can significantly affect soil archaeal abundance (Yang et al. 2014). Moisture, pH, and soil EC were considered as possible determinants limiting the abundance of soil (Hu et al. 2019a; Liu et al. 2017) [37,38]. However, in this study, Spearman correlation analysis showed that moisture was not a determinant of soil archaebacterial abundance in desert soil of PV parks. The insignificant role of $\mathrm{pH}$ in regulating archaeal community abundance in this study may be attributed to the small variation in soil $\mathrm{pH}$ across sites. The lack of correlation between archaeal abundance and EC may be due to high soil salinity levels that exceeded the migration threshold of the archaeal community in response to salinity changes (Hu et al. 2019a).

Diversity and Community Composition of Archaea in Desert Soils. Despite some variation within archaeal communities, they had low diversity in the desert soil of the PV park in this study (Fig. 4), a finding consistent with previous studies (Hu et al. 2019a; Nan et al. 2020). Low water usage, high evapotranspiration, high temperature variability, high salinity, lack of vegetation, low decomposition rates, and high soil $\mathrm{pH}$ are general trends in desert biomes, all of which may affect the proliferation of archaea, resulting in less diversity than bacterial and fungal communities. We did not find significant differences in archaeal alpha diversity when compared to bare ground soil outside the PV park, likely because the PV park was built recently. Furthermore, as a vehicle for archaeal activity, it takes a long time for the soil environment and vegetation to evolve in the study area; thus, it is unlikely that the diversity of archaea in the comparison sample sites would show significant differences in a short period of time. As for the PV panels themselves, the installation height and rotation angle of the panels may impact the richness of the vegetation in the field area, which in turn impacts the development of soil OM, which is a carrier of microbial growth.

To date, there is no consensus on the dominant archaeal phylum in different environments (Anish et al. 2015; Auguet et al. 2010; Huang et al. 2019; Li et al. 2021; Liu et al. 2009; Zhao et al. 2020). Several previous studies have documented changes in soil archaeal community structure with elevation, latitude, and sampling time (Liu et al. 2018; Shi et al. 2016; Wang et al. 2015b). Different geographic features may have different archaeal community structures, which is consistent with the results observed in the current study. In our study, the archaeal communities in all samples from areas $>50 \mathrm{~km}^{2}$ consisted mainly of Thaumarchaeota and Euryarchaeota, with Thaumarchaeota being much more dominant than Euryarchaeota, It is similar to the pattern observed in the Tibetan Plateau and other soils (Brochier-Armanet et al. 2008; Shi et al. 2016). Thaumarchaeota is a mesophilic group of archaea that is widespread in a variety of terrestrial and aquatic environments, and as the third phylum of archaea recognized in 2008 (Brochier-Armanet et al. 2008), most soil Thaumarchaea have ammonia oxidation capacity (Auguet et al. 2012; Pedneault et al. 2014) and play an important role in the global nitrogen and carbon cycles. It was found that the ammonia oxidation activity of Thaumarchaea even exceeds that of bacteria (Ke et al. 2014). The relative 
abundance of Thaumarchaea in the Gonghe PV Park was significant compared to other desert soil, and this increased abundance may be determined by the microbial ecological niche under extreme environmental stress. Meanwhile, the genus of Nitrososphaera under Thaumarchaea acts as an important ammonia oxidizer, using $\mathrm{CO}_{2}$ as the only carbon source and exhibiting strong autotrophic activity in the soil (Tourna et al. 2011). Nitrososphaera has been shown to be pro-alkaline(Tripathi et al. 2017). The dominant phylum, class, order, and genus of soil archaeal communities are consistent under different PV panel types in the desert zone. The taxonomic structure is simple and not significantly different. The similarity in structure may be mainly due to the similarity in relative abundance of the constituent taxa. However, some sub-taxa were found in specific PV panel types; therefore, it can be inferred that soil microbial communities differed among PV panel types due to the variation in PV panels and their surroundings. In addition, since higher vegetation coverage due to PV panel construction may improve the harsh environmental conditions of the original desert area, plants may indirectly affect the archaeal community through their impact on the physical environment of their root area. Various organic compounds released by various plant roots may also cause changes in soil archaeal communities (Ayangbenro and Babalola 2020).

Factors controlling the structure of soil archaeal communities. The factors affecting the distribution of soil archaea remain unknown (Huang et al. 2019; Meng et al. 2019; Shi et al. 2016). Previous studies have suggested that the structure of archaeal community in desert soil may be shaped by a combination of soil physical properties, nutrient content, heavy metal disturbance, plant root action, and pollution (Nan et al. 2020; Wang et al. 2019; Wang et al. 2011; Zhao et al. 2020). In this study, the combined results of RDA analysis and VPA suggest that a combination of many environmental variables and anthropogenic disturbances determine the distribution patterns of archaeal community structure in desert soil. Of all the factors we studied, soil conductivity received more attention because of its significant influence on archaeal community composition and diversity patterns. Soil conductivity serves as an equivalent indicator of salinity, which previous studies have found to be the most important environmental factor controlling the global distribution of archaea, and increased conductivity may reduce soil respiration and strongly affect archaeal community composition (Nan et al. 2020; Zhao et al. 2020). The strong explanatory power of several measurable environmental variables in predicting community composition in the current small-scale archaeal studies may reflect the importance of many soil and vegetation factors in influencing archaeal ecology and the dominant influence of only a few key factors. In the case of archaea, the residual spatial variation unexplained by measured environmental factors is often greater in other studies, leaving more room for dispersal-limiting effects in archaeal microbial geography. However, this unexplained variation simply reflects the spatial variability of unknown environmental factors (Shi et al. 2016; Wei et al. 2020). In future studies, in addition to soil factors, the climate of the Tibetan Plateau, where the study area is located, should also be considered. The influence of climate can be explained at regional and district scales. Regionally, the harsh climatic conditions make the Tibetan Plateau a unique microbial geographic distribution area.

\section{Conclusions}

In this study, we reported for the first time the characteristics of soil archaeal communities in a PV park in Qinghai Gonghe and explored the effects of PV park construction on archaea. The abundance of soil archaeal communities was lower in the PV park than outside the park, and soil archaeal abundance was closely related to OC and OM. At the taxonomic level, Thaumarchaeota (99.84\%) was dominant in the PV park, while Nitrososphaera was dominant at the genus level. The structure of soil archaeal communities was similar and did not show a significant difference between inside and outside of the park, or among different types of PV panels. The analysis showed that total carbon, alkaline decomposition nitrogen, and OM were the main factors affecting the alpha diversity of soil archaea, and soil water content was the main environmental factor affecting the phylum of Thaumarchaeota and the genus of Nitrososphaera. Quantitative VPA showed that soil physicochemical characteristics, vegetation growth condition, and PV panel installation parameters explained $33.67 \%, 25.77 \%$, and $7.02 \%$ of the observed variation. 


\section{ETHICS APPROVAL AND CONSENT TO PARTICIPATE}

Any submission that has data collected from human subjects requires ethics approval.

\section{CONSENT FOR PUBLICATION}

The authors of Wei Wu, Bo Yuan, Shengjuan Yue, Penghui Zou, Ruoting Yang, and Xiaode Zhou declare that they all agreed to publish.

\section{AVAILABILITY OF DATA AND MATERIAL}

The authors declare that [the/all other] data supporting the findings of this study are available within the article [and its supplementary information files].

\section{CONFLICT OF INTEREST}

The authors of Wei Wu, Bo Yuan, Shengjuan Yue, Penghui Zou, Ruoting Yang, and Xiaode Zhou declare that the research was conducted in the absence of any commercial or financial relationships that could be construed as a potential conflict of interest.

\section{FUNDING}

This work was financially supported by National Natural Science Foundation of China (No. 51979222).

\section{AUTHOR CONTRIBUTIONS}

W.W., B.Y., and X.Z. designed the study; B.Y., W.W., and S.Y. performed the study; B.Y., S.Y., R.Y., and P.Z. proposed the data analysis strategy; B.Y., and S.Y. analyzed the data; B.Y. assisted with the analytic tools; W.W., B.Y., wrote the original draft. All authors have read and agreed to the published version of the manuscript.

\section{ACKNOWLEDGEMENTS}

We would like to thank Editage (www.editage.cn) for English language editing.

\section{AUTHORS' INFORMATION}

Wei Wu: Doctor, lecturer, Xi'an University of Technology

Bo Yuan: Doctor, lecturer, Xi'an University of Technology

Shengjuan Yue: Doctor of Xi'an University of technology, lecturer of Qinghai University

Penghui Zou: Senior engineer of State Power Investment Group Qinghai Photovoltaic Industry Innovation Center Co., Ltd.

Ruoting Yang: Engineer of State Power Investment Group Qinghai Photovoltaic Industry Innovation Center Co., Ltd.

Xiaode Zhou: Professor and doctoral supervisor of Xi'an University of Technology

\section{References}

1. Anish B, Shekhar MN, Pijush B, Shayantan M, Debojyoti R, Sudip N, Anwesha H, Dhrubajyoti C, Suparna M, Maitree B (2015) Diversity and Distribution of Archaea in the Mangrove Sediment of Sundarbans. Archaea-an International Microbiological Journal 2015:968582

2. Auguet JC, Barberan A, Casamayor EO (2010) Global ecological patterns in uncultured Archaea. Isme Journal $4(2): 182-190$

3. Auguet JC, Triadó-Margarit X, Nomokonova N, Camarero L, Casamayor EO (2012) Vertical segregation and phylogenetic characterization of ammonia-oxidizing Archaea in a deep oligotrophic lake. Isme Journal 6(9):17861797 
4. Ayangbenro AS, Babalola OO (2020) Reclamation of arid and semi-arid soils: The role of plant growth-promoting archaea and bacteria - ScienceDirect. Current Plant Biology

5. Bates ST, Berg-Lyons D, Caporaso JG, Walters WA, Knight R, Fierer N (2011) Examining the global distribution of dominant archaeal populations in soil. The ISME Journal 5(5):908-917 doi:10.1038/ismej.2010.171

6. Brochier-Armanet C, Boussau B, Gribaldo S, Forterre P (2008) Mesophilic crenarchaeota: proposal for a third archaeal phylum, the Thaumarchaeota. Nature Reviews Microbiology 6

7. Dai L, Liu C, Yu L, Song C, Peng L, Li X, Tao L, Li G (2018) Organic Matter Regulates Ammonia-Oxidizing Bacterial and Archaeal Communities in the Surface Sediments of Ctenopharyngodon idellus Aquaculture Ponds. Frontiers in Microbiology 9 doi:10.3389/fmicb.2018.02290

8. Ebhota WS, Jen T-C (2020) Fossil Fuels Environmental Challenges and the Role of Solar Photovoltaic Technology Advances in Fast Tracking Hybrid Renewable Energy System. International Journal of Precision Engineering and Manufacturing-Green Technology 7(1):97-117 doi:10.1007/s40684-019-00101-9

9. Feng K, Zhang Z, Cai W, Liu W, Xu M, Yin H, Wang A, He Z, Deng Y (2017) Biodiversity and species competition regulate the resilience of microbial biofilm community. Molecular Ecology 26

10. Gao X, Yang L, Lyu F, Ma L, Hui X, Hou X, Li H (2016) EFFECT OF PV FARM ON SOIL TEMPERATURE IN GOLMUD DESERT AREA. Acta Energiae Solaris Sinica 37(6):1439-1445

11. Hu AY, Wang HJ, Li JW, Liu J, Chen NW, Yu CP (2016) Archaeal community in a human-disturbed watershed in southeast China: diversity, distribution, and responses to environmental changes. Applied Microbiology and Biotechnology 100(10):4685-4698 doi:10.1007/s00253-016-7318-X

12. Hu Y, Zhang Z, Huang L, Qi Q, Liu L, Zhao Y, Wang Z, Zhou H, Lv X, Mao Z (2019a) Shifts in soil microbial community functional gene structure across a 61-year desert revegetation chronosequence. Geoderma 347:126-134

13. Hu Y, Zhang Z, Huang L, Qi Q, Liu L, Zhao Y, Wang Z, Zhou H, Lv X, Mao Z, Yang Y, Zhou J, Kardol P (2019b) Shifts in soil microbial community functional gene structure across a 61-year desert revegetation chronosequence. Geoderma 347:126-134 doi:https://doi.org/10.1016/j.geoderma.2019.03.046

14. Huang M, Chai L, Jiang D, Zhang M, Zhao Y, Huang Y (2019) Increasing aridity affects soil archaeal communities by mediating soil niches in semi-arid regions. Sci Total Environ 647:699-707 doi:https://doi.org/10.1016/j.scitotenv.2018.07.305

15. Jiang H, Dong H, Yu B, Liu X, Li Y, Ji S, Zhang CL (2007) Microbial response to salinity change in Lake Chaka, a hypersaline lake on Tibetan plateau. Environmental Microbiology 9(10):2603-2621

16. Ke X, Lu Y, Conrad R (2014) Different behaviour of methanogenic archaea and Thaumarchaeota in rice field microcosms. Fems Microbiology Ecology 87(1)

17. Li F, Yang Y, Zhao J, Chen Z, Gao X, Shen Y (2019) Review on Energy Impact of Photovoltaic Power Station Construction and Operation on Climate and Environment. Advances in Meteorological Science and Technology 9(2):71-77

18. Li M, Mi T, He H, Chen Y, Zhen Y, Yu Z (2021) Active bacterial and archaeal communities in coastal sediments: Biogeography pattern, assembly process and co-occurrence relationship. Sci Total Environ 750:142252 doi:https://doi.org/10.1016/j.scitotenv.2020.142252

19. Li S, Gao Q, Wang X, Lan L, Yang Z (2016) Characteristics of Vegetation and Soil Property Changes by Photovoltaic Plant Interference in Alpine Desert Steppe \%J Journal of Soil and Water Conservation. Journal of Soil and Water Conservation 30(6):325-329

20. Li W, Zhang S, Zhang Q, Dong C (2007) Rapid prediction of available N, P and K content in soil using near-infrared reflectance spectroscopy. Transactions of the Chinese Society of Agricultural Engineering 23:55-59

21. Liu L, Liu Y, Hui R, Xie M (2017) Recovery of microbial community structure of biological soil crusts in successional stages of Shapotou desert revegetation, northwest China. Soil Biology \& Biochemistry

22. Liu P, Klose M, Conrad R (2019) Temperature-Dependent Network Modules of Soil Methanogenic Bacterial and Archaeal Communities. Other 10 
23. Liu R, Zhang Y, Ding R, Li D, Gao Y, Yang M (2009) Comparison of archaeal and bacterial community structures in heavily oil-contaminated and pristine soils. Journal of Bioscience and Bioengineering 108(5):400-407 doi:https://doi.org/10.1016/j.jbiosc.2009.05.010

24. Liu Y, Li X, Xing Z, Zhao X, Pan Y (2013) Responses of soil microbial biomass and community composition to biological soil crusts in the revegetated areas of the Tengger Desert. Applied Soil Ecology 65:52-59

25. Liu Y, Zhao L, Wang Z, Liu L, Zhang P, Sun J, Wang B, Song G, Li X (2018) Changes in functional gene structure and metabolic potential of the microbial community in biological soil crusts along a revegetation chronosequence in the Tengger Desert. Soil Biology and Biochemistry 126:40-48 doi:https://doi.org/10.1016/j.soilbio.2018.08.012

26. Luvizott DM, Araujo JE, Silva MDCP, Dias ACF, Kraft B, Tegetmeye H, Strous M, Andreote FD (2019) The rates and players of denitrification, dissimilatory nitrate reduction to ammonia (DNRA) and anaerobic ammonia oxidation (anammox) in mangrove soils. Anais Da Academia Brasileira De Ciencias 91 doi:10.1590/0001-3765201820180373

27. Meng, Wang, Shibao, Chen, Li, Chen, Duo, Wang (2019) Saline stress modifies the effect of cadmium toxicity on soil archaeal communities. Ecotoxicology \& Environmental Safety 182:109431-109431

28. Nan L, Guo Q, Cao S (2020) Archaeal community diversity in different types of saline-alkali soil in arid regions of Northwest China. Journal of Bioscience \& Bioengineering

29. Nelson DW, Sommers L, Page AL, Miller RH, Keeney DR (1982) Total carbon, organic carbon, and organic matter. Methods of Soil Analysis 9:539-552

30. Nelson DW, Sommers LE (1973) Determination of Total Nitrogen in Plant Material. Agronomy Journal 65(1)

31. Nižetić S, Giama E, Papadopoulos AM (2018) Comprehensive analysis and general economic-environmental evaluation of cooling techniques for photovoltaic panels, Part II: Active cooling techniques. Energy Conversion and Management 155:301-323 doi:https://doi.org/10.1016/j.enconman.2017.10.071

32. Pedneault E, Galand PE, Potvin M, Tremblay JÉ, Lovejoy C (2014) Archaeal amoA and ureC genes and their transcriptional activity in the Arctic Ocean. Scientific Reports 4 doi:10.1038/srep04661

33. Pires ACC, Cleary DFR, Almeida A, Cunha A, Dealtry S, Mendonca-Hagler LCS, Smalla K, Gomes NCM (2012) Denaturing Gradient Gel Electrophoresis and Barcoded Pyrosequencing Reveal Unprecedented Archaeal Diversity in Mangrove Sediment and Rhizosphere Samples. Applied \& Environmental Microbiology 78(16):5520

34. Rothrock MJ, Jr., Garcia-Pichel F (2005) Microbial diversity of benthic mats along a tidal desiccation gradient. Environ Microbiol 7(4):593-601 doi:10.1111/j.1462-2920.2005.00728.x

35. Rui J, Li J, Wang S, An J, Liu WT, Lin Q, Yang Y, He Z, Li X (2015) Responses of Bacterial Communities to Simulated Climate Changes in Alpine Meadow Soil of the Qinghai-Tibet Plateau. Appl Environ Microbiol 81(17):6070-6077

36. Shi Y, Adams JM, Ni Y, Yang T, Jing X, Chen L, He JS, Chu H (2016) The biogeography of soil archaeal communities on the eastern Tibetan Plateau. Scientific Reports 6

37. Sorokin DY, Messina E, Smedile F, Roman P, Damsté JSS, Ciordia S, Mena MC, Ferrer M, Golyshin PN, Kublanov IV (2017) Discovery of anaerobic lithoheterotrophic haloarchaea, ubiquitous in hypersaline habitats. Isme Journal 11(5):1245-1260

38. Tawalbeh M, Al-Othman A, Kafiah F, Abdelsalam E, Almomani F, Alkasrawi M (2021) Environmental impacts of solar photovoltaic systems: A critical review of recent progress and future outlook. Sci Total Environ 759:143528 doi:https://doi.org/10.1016/j.scitotenv.2020.143528

39. Tourna M, Stieglmeier M, Spang A, Martin K, Schintlmeister A, Urich T, Engel M, Schloter M, Wagner M, Richter A (2011) Nitrososphaera viennensis an ammonia oxidizing archaeon from soil. Proceedings of the National Academy of Sciences of the United States of America 108(20):p.8420-8425

40. Tripathi BM, Moroenyane I, Sherman C, Lee YK, Adams JM, Steinberger Y (2017) Trends in Taxonomic and Functional Composition of Soil Microbiome Along a Precipitation Gradient in Israel. Microb Ecol 74(1):168-176 doi:10.1007/s00248-017-0931-0

41. Turney D, Fthenakis V (2011) Environmental impacts from the installation and operation of large-scale solar power 
plants. Renewable and Sustainable Energy Reviews 15(6):3261-3270 doi:https://doi.org/10.1016/j.rser.2011.04.023

42. Wang J-T, Cao P, Hu H-W, Li J, Han L-L, Zhang L-M, Zheng Y-M, He J-Z (2015a) Altitudinal Distribution Patterns of Soil Bacterial and Archaeal Communities Along Mt. Shegyla on the Tibetan Plateau. Microb Ecol 69(1):135-145 doi:10.1007/s00248-014-0465-7

43. Wang JT, Cao P, Hu HW, Li J, Han LL, Zhang LM, Zheng YM, He JZ (2015b) Altitudinal Distribution Patterns of Soil Bacterial and Archaeal Communities Along Mt. Shegyla on the Tibetan Plateau. Microb Ecol 69(1):135-145 doi:10.1007/s00248-014-0465-7

44. Wang N, Wang A, Xie J, He M (2019) Responses of soil fungal and archaeal communities to environmental factors in an ongoing antimony mine area. Sci Total Environ 652:1030-1039 doi:10.1016/j.scitotenv.2018.10.300

45. Wang X, Zhen H, Bai Z, Tang J, Ma A, Jizheng H, Zhuang G (2011) Archaeal community structure along a gradient of petroleum contamination in saline-alkali soil. Journal of environmental sciences 23(11):1858-1864

46. Wei G, Li M, Shi W, Tian R, Gao Z (2020) Similar drivers but different effects lead to distinct ecological patterns of soil bacterial and archaeal communities. Soil Biology \& Biochemistry 144:107759

47. Wilberforce T, Baroutaji A, El Hassan Z, Thompson J, Soudan B, Olabi AG (2019) Prospects and challenges of concentrated solar photovoltaics and enhanced geothermal energy technologies. Sci Total Environ 659:851-861 doi:https://doi.org/10.1016/j.scitotenv.2018.12.257

48. Yang Y, Gao Y, Wang S, Xu D, Yu H, Wu L, Lin Q, Hu Y, Li X, He Z, Deng Y, Zhou J (2014) The microbial gene diversity along an elevation gradient of the Tibetan grassland. The ISME Journal 8(2):430-440 doi:10.1038/ismej.2013.146

49. Zhao L, Liu Y, Yuan S, Li Z, Sun J, Li X (2020) Development of archaeal communities in biological soil crusts along a revegetation chronosequence in the Tengger Desert, north central China. Soil and Tillage Research 196:104443 doi:https://doi.org/10.1016/j.still.2019.104443 


\section{Supplementary Files}

This is a list of supplementary files associated with this preprint. Click to download.

- SupplementaryFiguresandTables.docx 